\title{
Hyperspectral imaging with deformable gratings fabricated with metal-elastomer nanocomposites
}

Marco A. C. Potenza, Daniele Nazzari, Llorenç Cremonesi, Ilaria Denti, and Paolo Milani

Citation: Review of Scientific Instruments 88, 113105 (2017); doi: 10.1063/1.5009180

View online: https://doi.org/10.1063/1.5009180

View Table of Contents: http://aip.scitation.org/toc/rsi/88/11

Published by the American Institute of Physics

\section{Articles you may be interested in}

True randomness from an incoherent source

Review of Scientific Instruments 88, 113101 (2017); 10.1063/1.4986048

Coincidence velocity map imaging using Tpx3Cam, a time stamping optical camera with $1.5 \mathrm{~ns}$ timing resolution

Review of Scientific Instruments 88, 113104 (2017); 10.1063/1.4996888

A versatile apparatus for two-dimensional atomtronic quantum simulation

Review of Scientific Instruments 88, 113102 (2017); 10.1063/1.5009584

A high precision flat crystal spectrometer compatible for ultra-high vacuum light source

Review of Scientific Instruments 88, 113108 (2017); 10.1063/1.5010995

Aspheric optical surface profiling based on laser scanning and auto-collimation

Review of Scientific Instruments 88, 113106 (2017); 10.1063/1.4995685

Saturation of the photoneutralization of $\mathrm{H} \mathrm{H}^{-}$beam in continuous operation

Review of Scientific Instruments 88, 113103 (2017); 10.1063/1.4995390

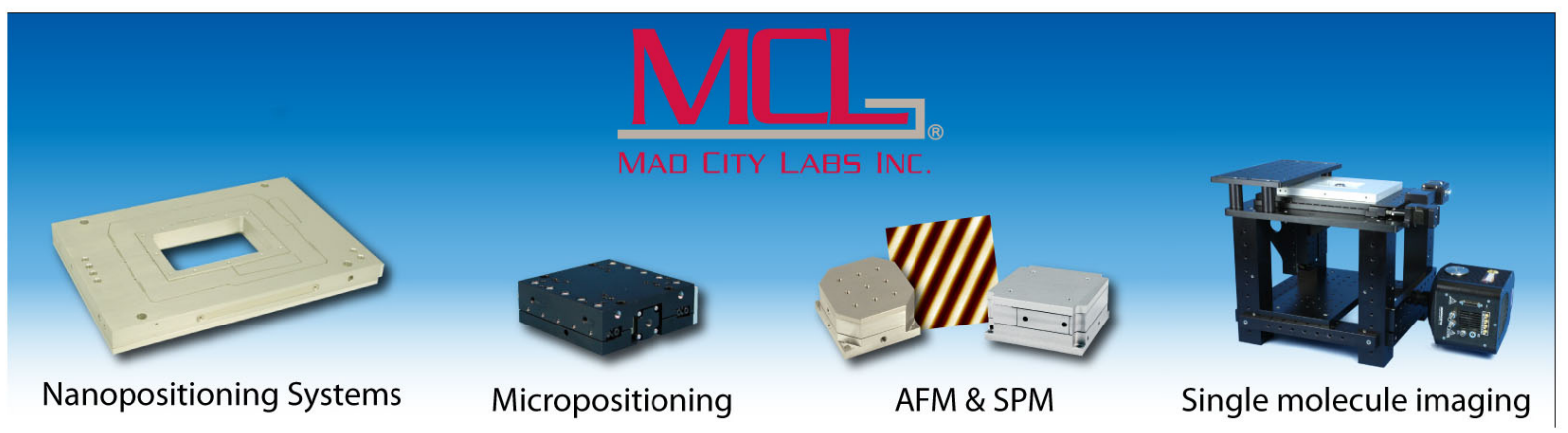




\title{
Hyperspectral imaging with deformable gratings fabricated with metal-elastomer nanocomposites
}

\author{
Marco A. C. Potenza, ${ }^{\text {a) }}$ Daniele Nazzari, Llorenç Cremonesi, Ilaria Denti, and Paolo Milania) \\ CIMAINA and Dipartimento di Fisica, Università degli Studi di Milano, Via Celoria 16, 20133 Milano, Italy
}

(Received 15 September 2016; accepted 10 October 2017; published online 9 November 2017)

\begin{abstract}
We report the fabrication and characterization of a simple and compact hyperspectral imaging setup based on a stretchable diffraction grating made with a metal-polymer nanocomposite. The nanocomposite is produced by implanting Ag clusters in a poly(dimethylsiloxane) film by supersonic cluster beam implantation. The deformable grating has curved grooves and is imposed on a concave cylindrical surface, thus obtaining optical power in two orthogonal directions. Both diffractive and optical powers are obtained by reflection, thus realizing a diffractive-catoptric optical device. This makes it easier to minimize aberrations. We prove that, despite the extended spectral range and the simplified optical scheme, it is actually possible to work with a traditional CCD sensor and achieve a good spectral and spatial resolution. Published by AIP Publishing. https://doi.org/10.1063/1.5009180
\end{abstract}

\section{INTRODUCTION}

Hyperspectral imaging (HSI) is a technology where traditional optical or infrared imaging is combined with spectroscopic analysis of any particular object or landscape. This is often obtained by using diffraction optics that disperse different wavelengths of light, together with sensors able to capture and to build a wavelength-intensity map of a scene with a very high spatial resolution. ${ }^{1}$ Each pixel of an image is analyzed in terms of its electromagnetic spectrum, in order to generate a set of "images" each corresponding to a narrow spectral window (data cube). The combination of spectral data and spatial details allows the high-speed analysis of the chemical content, product uniformity and quality, and a host of other spectral characteristics and attributes. ${ }^{2}$

Recent computing advancements and improvements in data acquisition and analysis have extended HSI to food inspection, pathogen detection, airport security, law enforcement, and oil and gas pipeline inspection. ${ }^{3,4}$ However, the widespread use of hyperspectral technology is limited by its relatively high costs in terms of hardware. Due to the growing demand for the deployment of HSI-based devices in remotesensing applications, a huge effort is concentrated in hardware miniaturization and cost reduction. ${ }^{5}$

A typical dispersive HSI device is composed of a collection optics, a grating, and the optical elements needed to form spectral images onto the detector. Usually, the data cube is obtained by imaging the spectral distribution of a thin slice of the object and by scanning the object perpendicularly to the slice direction. Depending on the spectral and spatial resolution requirements, the optical setup can be very complex with a high number of components, making it hard to obtain compact, lightweight, and low-cost devices. To this purpose, the reduction of optical components and the simplification of the optical scheme are thus

a)Authors to whom correspondence should be addressed: marco.potenza@ unimi.it and paolo.milani@unimi.it. a strategic goal for the fabrication of low-cost HSI-based sensors. ${ }^{1}$

The interest for curved gratings (concave or convex) has considerably increased in view of applications where the number of optical elements needs to be reduced, especially considering the increasing demand of miniaturization. ${ }^{6}$ High-resolution curved diffraction gratings are fabricated with different methods such as ruling engines: this is a slow and delicate process requiring a careful control on the mechanics of the engine and on external vibrations. ${ }^{7}$ Because of the difficulties in this process, most of the gratings adopted in instrumental applications are high quality, more affordable replicas of the ruled master gratings. Alternative fabrication methods are based on the interference fringe field in a photoresist to create a holographic grating ${ }^{8}$ or subtractive technologies such as electron beam lithography, ${ }^{9}$ ion beam etching, ${ }^{10}$ and laser ablation. Such methods are very effective, although expensive and requiring complex hardware. ${ }^{11,12}$

In order to fabricate low-cost optical devices, deformable optical elements (lenses, mirrors, and gratings) have been proposed as effective ingredients of compact, inexpensive, and portable solutions based on adaptive optics. ${ }^{13-15}$ In particular, the use of tunable gratings based on stretchable reflective substrates could revolutionize the design of optical mini spectrometers and significantly widen their field of applications. ${ }^{16}$ Although appealing, the use of deformable reflecting optical elements has been hampered by the difficulty of integrating reflecting metallic films on elastomeric substrates due to delamination of the metallic layer upon deformation. ${ }^{14}$

We recently reported the fabrication of stretchable reflective gratings based on metal-elastomer nanocomposites obtained by Supersonic Cluster Beam Implantation (SCBI) in polydimethylsiloxane (PDMS). ${ }^{16}$ The optical quality of these components has proven to be stable against cyclical deformation and stretching.

Here we report the proof of the concept of the use of these highly deformable gratings for the fabrication of a simple and cheap hyperspectral camera. Thanks to their deformability, 
optical power can be arbitrarily imposed on nanocomposite gratings in a simple and effective way by sticking them on cylindrical substrates. Indeed, by sticking gratings with proper circular grooves on concave cylindrical surfaces, it is possible to impose optical power in the direction perpendicular to the axis of symmetry of the cylinder, thus achieving focalization in this direction. In such a way, astigmatic wavefronts can be obtained at will, the stigmatic case being just the particular case exploited here. Compared to rigid curved gratings, our solution achieves a smaller spectral resolution; on the other hand, it is much less demanding in terms of fabrication complexity and costs.

\section{EXPERIMENTAL SECTION}

\section{A. Hyperspectral apparatus}

The experimental apparatus is sketched in Fig. 1. The white light from a halogen lamp (USHIO, mod. EKE, $150 \mathrm{~W}$ ) transilluminates the sample. In order to minimize chromatic aberrations, light is collected by two-faced doublets that image the sample onto the plane on which the slit lies. The magnification of the system is 1.14 . A slit $0.3 \mathrm{~mm}$ wide is placed in the focal plane of a $f=75 \mathrm{~mm}$ collecting achromatic doublet, the input effective $f$-number being about 7.5 although the specific illumination limits the angular spread to double this value (see Subsection 2 of the Appendix). A parallel beam with a diameter of $10 \mathrm{~mm}$ is sent onto the grating, and the first order diffracted light is then collected by a CCD sensor where a wavelength dispersed image of the slit is obtained, as described below. The CCD is a black and white Atmel full-frame sensor with $3500 \times 2300$ square pixels with a side of $10 \mu \mathrm{m}$ and a 12-bit digitization. An external shutter has been placed along the optical axis before the slit. The position of the sensor has been properly chosen, as discussed below.

The deformable elastomeric grating is stuck onto a concave cylindrical plastic surface, with a radius $R=230 \mathrm{~mm}$ and the axis of symmetry in the vertical direction. This determines the horizontal focusing of the system, while the vertical focusing is given by the curved shape of the grooves. These are oriented in such a way that the center of curvature is placed at the same vertical position of the impinging beam. Their orientation guarantees that the light is properly focused by diffraction from the curved grooves in the vertical direction, thus forming an image of the slit onto the sensor at any wavelength. Therefore, for any vertical slice of the sample, a two dimensional (2D) picture is recorded, containing the space-wavelength information of the given slice of the sample. A translation stage displaces the sample transversely to the slit length, with spatial increments smaller than the slit aperture (see Sec. II C). In such a way, the complete spacewavelength information is obtained for the whole sample, and images can be recovered for each wavelength through a simple algorithm.

\section{B. Deformable grating fabrication and characterization}

Transparent circular gratings were fabricated by making a polydimethylsiloxane (PDMS) replica from a master consisting of a commercial DVD (groove pitch $740 \mathrm{~nm}$ ). The mold was sliced in rectangles of $40 \mathrm{~mm} \times 25 \mathrm{~mm}$, using the radius of the circular gratings as the main rectangle axis. The PDMS liquid precursor (Sylgard 184 Silicone Elastomer Kit from Dow Corning, mixed in a 10:1 ratio) was cast on the DVD slices and subsequently spin coated at $800 \mathrm{rpm}$ for $40 \mathrm{~s}$, reaching the thickness of about $200 \mu \mathrm{m}$ and finally crosslinked at room temperature for $48 \mathrm{~h}$. After cross-linking, the mold was carefully detached from the master (Fig. 2).

The transparent PDMS gratings were then made reflective by implanting silver nanoparticles using a Supersonic Cluster Beam Implantation (SCBI) apparatus equipped with a pulsed microplasma cluster source (PMCS), as described in detail in Ref. 15.

Briefly, a PMCS consists in a ceramic body with a cavity in which a solid silver target (purity 99.9\%) is vaporized by a localized electrical discharge supported by a pulsed injection of an inert gas (He or Ar) at high pressure (40 bars).
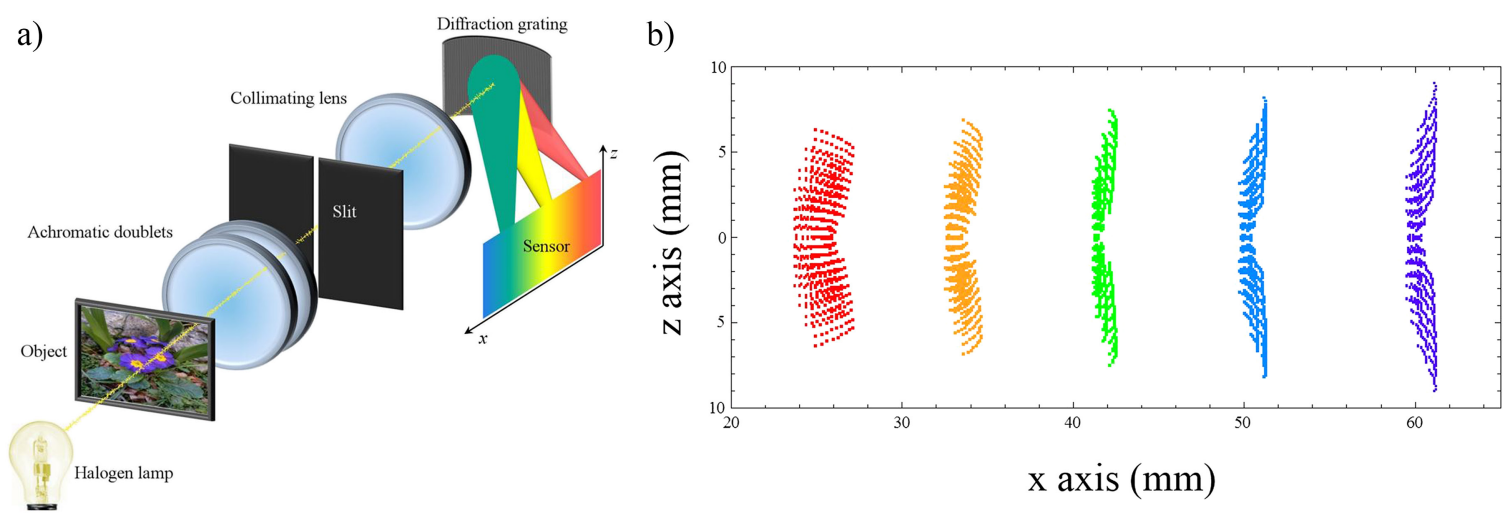

FIG. 1. (a) Schematic representation of the experimental apparatus (not to scale). The white light from a halogen lamp illuminates the sample and two achromatic doublets form an image onto the slit plane minimizing aberrations. The light from the slit is collimated by an achromatic doublet and sent onto a curved nanocomposite grating that imposes the necessary optical power to the diffracted wavefronts. Light is collected onto the plane surface of a CCD sensor parallel to the optical axis (the rainbow-colored plane). (b) Ray-tracing results representing the formation of the spectral lines upon the sensor plane, as discussed below (see Subsection 2 of the Appendix). From right to left, wavelengths are 450, 500, 550, 600, $650 \mathrm{~nm}$. 


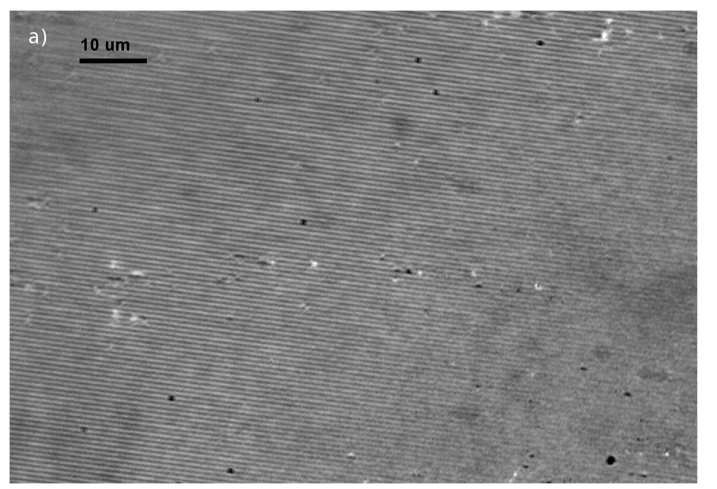

b)

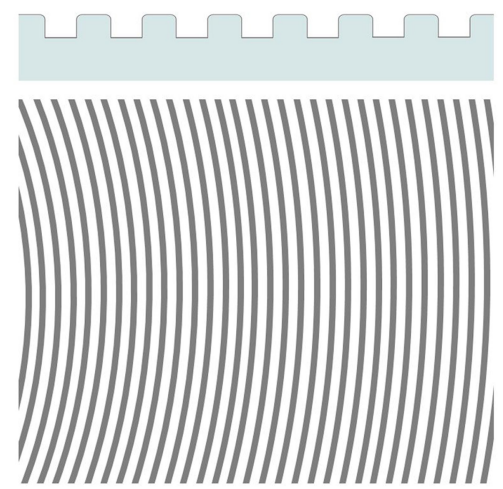

FIG. 2. (a) Optical microscope image of the PDMS diffraction grating, taken after the implantation procedure with a $100 \times$ objective. The implantation process has not warped the grooves, previously obtained on the PDMS with a hard grating that served as a mold. (b) Schematic representation of the circular grooves and cross-sectional profile.

The metal atoms, sputtered from the target, aggregate in the source cavity to form metal clusters; the mixture of clusters and inert gas expands through a nozzle forming a supersonic beam into an expansion chamber kept at a pressure of about $10^{-6}$ mbar. Electrically neutral nanoparticles exiting the PMCS are aerodynamically accelerated in a highly collimated beam with divergence lower than $1^{\circ}$ and with a kinetic energy of roughly $0.5 \mathrm{eV} / \mathrm{atom}$. The central part of the cluster beam enters a second vacuum chamber (deposition chamber) through a skimmer, where the beam is intercepted by the polymeric substrate. The measure of the quantity of material implanted into the PDMS is obtained by placing a half-masked hard substrate (glass or silicon) next to the grating during the implantation process, in order to intercept the same amount of nanoparticles. The thickness of the cluster-assembled film deposited on the hard substrate corresponds to the equivalent thickness $t_{\mathrm{eq}}$ in the case of the metal-polymer nanocomposite. We metallized PDMS gratings with an equivalent thickness of approximately $60 \mathrm{~nm}$, at a deposition rate of about $0.08 \AA \times \mathrm{s}^{-1}$. This value of $t_{\text {eq }}$ provides a diffraction efficiency of some $10 \%$ across the entire visible spectrum. ${ }^{15,16}$

The gratings have been characterized independently in terms of the capability of curving the wavefronts and of producing small focal spots, as detailed in the Appendix. Here we just mention that the main limitation in the optical properties comes primarily from the limited surface quality of the gratings themselves. The presence of dust on the master can lead to the formation of local defects in the grating's pattern. A smooth surface is essential to ensure a homogenous adhesion of the grating on the cylinder.

Different gratings have been tested with comparable results. Measurements have been performed with a spatially filtered $\mathrm{He}-\mathrm{Ne}$ laser beam, expanded to a diameter of $10 \mathrm{~mm}$, as it is the case in our system. Beam shaping is as expected for both the cylindrical and the diffractive focusing although some limitations arise that prevent the system to reach a good spatial resolution. Quantitatively speaking, the beam follows the geometrical/Gaussian optics laws until the beam size goes approximately down to $100 \mu \mathrm{m}$. Limitations arise when the size of the beam is measured close to the focal region, where the beam does not narrow to what is expected from basic optics rules (a few microns in our case). This limitation may be linked to wavefront distortions imposed by the grating at a small length scale, as it is evident from highly modulated intensity distributions measured in the far field of the beam (Subsection 1 of the Appendix). Nevertheless, since we are not interested in pushing the resolution to the highest reachable level, this feature is not limiting our results here: as described in Sec. II C, the system is mainly limited by defocusing rather than diffraction.

\section{Hyperspectral image reconstruction: Theoretical and practical considerations}

Irrespective of the astigmatic or stigmatic optical power, working independently on the two perpendicular directions is the key point of this work. Indeed, cylindrical surfaces are unique since the metric of a cylinder is conformal to the Euclidean one. ${ }^{17}$ Therefore, spacing will be globally maintained irrespective of the local orientation of the grooves, including the case of interest here of circular, equally spaced grooves. On the contrary, any deformable grating stuck onto a curved surface will inherit the corresponding metric, thus changing locally the diffraction properties. We stress here that cylindrical surfaces also guarantee that the nanocomposite is not affected by any stress, strain, elongation, etc., the only need being to slightly bend it. In principle, by exploiting this approach, high optical power can be imposed on the gratings. Some drawbacks are encountered, mainly related to the current realization of the gratings, as it will be discussed below.

Several requirements have been identified to reconstruct hyperspectral images with a system that essentially needs no optical components other than the grating. First, the vertical (V) and horizontal $(\mathrm{H})$ focusing conditions must be matched by adjusting the curvature of the grating together with the radius of its circular grooves. Second, the defocusing deriving from reading the light intensity focused onto a curved surface with a planar CCD sensor must be minimized, possibly without introducing any further optical elements. We describe here a condition that minimizes the drawbacks in view of performing hyperspectral imaging in the visible range and represents a proof of the concept of the use of elastomeric gratings to hyperspectral imaging. This is the focus here, to prove the feasibility of a hyperspectral camera without the need of several optical groups. Notice that the current performances of our mock-up are somehow limited with respect to a corresponding state-of-the-art device of a comparable size. As mentioned 


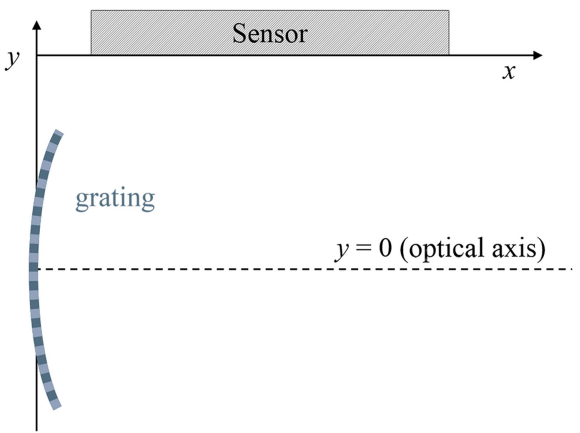

(a)

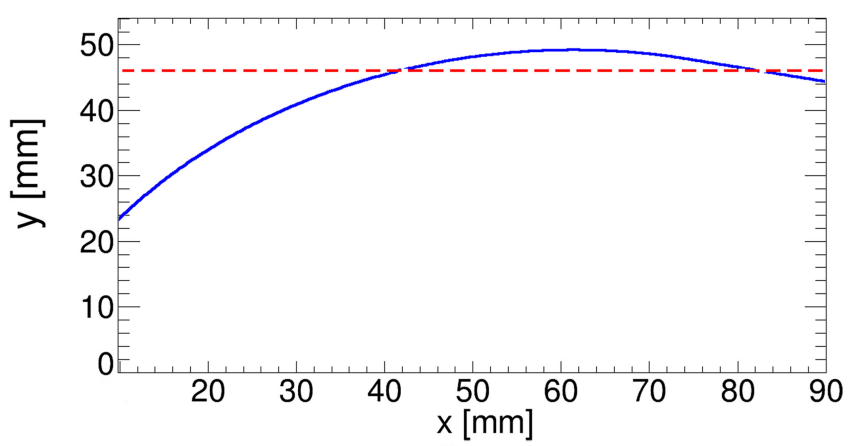

(b)

FIG. 3. (a) Reference frame adopted in Eqs. (1) and (2) (top view). (b) Comparison of the horizontal (solid, blue) and vertical (dashed, red) focusing according to Eqs. (1)-(4). The CCD sensor is placed in correspondence to the dashed red line, in the plane perpendicular to the figure. $y=0$ represents the optical axis as defined earlier. Wavelength decreases along the $x$ axis. This configuration minimizes the differences in focal distance within the wavelength range of interest here.

earlier and better discussed in the Appendix, this is mainly due to the surface quality of the gratings at this initial stage of development.

The parametric equations for the focal positions imposed in the $\mathrm{H}$ direction by the cylindrical surface can be written by simply using geometrical optics as ${ }^{15}$

$$
\begin{gathered}
x(\lambda)=\frac{R\left(1-\frac{\lambda^{2}}{d^{2}}\right)^{\frac{3}{2}}}{1+\sqrt{1-\frac{\lambda^{2}}{d^{2}}},} \\
y(\lambda)=\frac{R\left(1-\frac{\lambda^{2}}{d^{2}}\right)}{1+\sqrt{1-\frac{\lambda^{2}}{d^{2}}}} \cdot \frac{\lambda}{d},
\end{gathered}
$$

where $\lambda$ is the light wavelength, $d=740 \mathrm{~nm}$ is the groove spacing, $R=230 \mathrm{~mm}$ is the radius of the cylindrical surface, $x$ is the coordinate along the sensor, and $y$ is the orthogonal axis in the horizontal plane. The focal length in the $\mathrm{V}$ direction can be obtained on the basis of elementary Fourier optics ${ }^{18}$ and gives the following parametric equations:

$$
\begin{gathered}
y(\lambda)=r \sqrt{\frac{d^{2}}{\lambda^{2}}-1}, \\
y(\lambda)=r,
\end{gathered}
$$

where $r=46 \mathrm{~mm}$ is the radius of the circular grooves (measured on the plane grating) intercepted by the optical axis. We imposed the same focusing for the $\mathrm{H}$ and $\mathrm{V}$ directions to occur in the green at $546 \mathrm{~nm}$ (Hg emission line; see below). The radii of the cylinder $(R)$ and grooves $(r)$ have then been chosen to form an image of the slit onto the sensor. Finally, the CCD sensor has been placed in such a way to meet the $\mathrm{V}$ focusing condition, which can be found at a distance of $46 \mathrm{~mm}$ from the optical axis of the system. A magnification of a factor $m=0.83$ at $546 \mathrm{~nm}$ is obtained so that the image of the slit is $0.25 \mathrm{~mm}$ wide. This corresponds to a wavelength resolution of approximately $\Delta \lambda=2 \mathrm{~nm}$, as evaluated under ideal conditions. A plot of the magnification measured as a function of the wavelength is shown in the Appendix. When showing the results, we do not compensate for the changing magnification, this being just a rigid scaling of the images which does not affect the main results.

As it can be derived from the above relations, a crucial parameter is the groove spacing of the grating. It must be small enough to determine an effective dispersion and to effectively separate the diffracted beams. By contrast, a small spacing introduces a non-negligible dependence on wavelength, which becomes rampant in the horizontal direction when $\lambda \sim d$. Here we have adopted $d=740 \mathrm{~nm}$, which only limits the performances in the red portion of the spectrum, as it appears from the curves in Fig. 3. Apart from the unique wavelength of $546 \mathrm{~nm}$, any other wavelength will be focused out of the CCD plane according to Fig. 3, thus slightly reducing the wavelength resolution. To evaluate this effect, we have determined the size of the defocused slit image at the sensor surface. Simple geometrical optics arguments used to evaluate the $\mathrm{H}$ size of the defocused image of the entrance slit give the result shown in Fig. 4, where the width of the intensity distributions at the sensor plane is plotted against the wavelength $\lambda$. Moreover, a dedicated ray tracing code has been realized to describe the geometrical optics performances of the system, as detailed in Subsection 2 of the Appendix. Experimentally, we characterized the resolution of the system by illuminating the slit with a low pressure $\mathrm{Hg}-\mathrm{Ar}$ lamp and compared the measured size of the slit images formed onto the

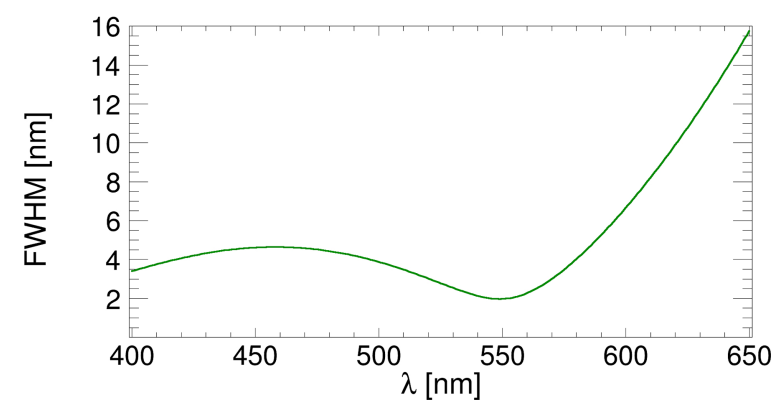

FIG. 4. Plot of the width of the slit image (converted in $\mathrm{nm}$ ) as a function of the wavelength (in nm), obtained as discussed in the text to keep into account the defocus due to the effect described by Fig. 3 . 
TABLE I. Comparison between measured and expected spectral resolution at three different wavelengths of the $\mathrm{Hg}$ lamp spectrum. Symbol * indicates that the line is actually the doublet at wavelengths $577 \mathrm{~nm}$ and $579 \mathrm{~nm}$. For the spectral resolution at wavelength $578 \mathrm{~nm}$, the theoretical value is indicated in round brackets, while the expected larger value is derived in the text.

\begin{tabular}{lcc}
\hline \hline$\lambda(\mathrm{nm})$ & $\Delta \lambda_{\text {meas }}(\mathrm{nm})$ & $\Delta \lambda_{\exp }(\mathrm{nm})$ \\
\hline 435 & 3.7 & 4.4 \\
546 & 3.2 & 2 \\
$578^{*}$ & 6.1 & $5.8(3.8)$ \\
\hline \hline
\end{tabular}

sensor to the expected values for the three $\mathrm{Hg}$ wavelengths: $\lambda_{1}=435 \mathrm{~nm}, \lambda_{2}=546 \mathrm{~nm}$, and $\lambda_{3}=578 \mathrm{~nm}$ (Ar lines lie outside the visible range). Notice that $\lambda_{3}$ is actually a doublet with wavelengths of $577 \mathrm{~nm}$ and $579 \mathrm{~nm}$. We will not be sensible to this small spacing although we will measure a broader line.

In Table I, we compare the expected resolution evaluated as discussed earlier to the measured ones at the three wavelengths of the lamp. In the first column, we report the wavelength $\lambda$, in the second column, the measured resolution $\Delta \lambda_{\text {meas }}$, expressed in terms of the FWHM of the slit images, and in the last one, the theoretical values $\Delta \lambda_{\exp }$ expected on the basis of the simple approach described earlier. Notice that the larger $\Delta \lambda_{\text {meas }}$ is obtained at $578 \mathrm{~nm}$, just for the doublet case. Therefore, we do not compare this number directly to the expected value obtained from Fig. 3 , that is, $\Delta \lambda_{\exp }=3.8 \mathrm{~nm}$ (reported in parentheses), but we estimate the broadening of the line on the basis of the known line spacing and by imposing the expected enlargement to both lines. The result is a width of approximately $5.8 \mathrm{~nm}$.

As mentioned earlier, each frame collected by the CCD sensor provides the hyperspectral image of one vertical slice of the sample. To image the whole sample surface, we translate it transversely with respect to the optical axis, thus collecting a stack of 360 hyperspectral images of slices that covers the entire sample. The translation step has been chosen to be $50 \mu \mathrm{m}$ long, smaller than the slit aperture $(300 \mu \mathrm{m})$, which determines the horizontal spatial resolution of the system. The vertical resolution has been measured as a function of the wavelength, by imaging thin horizontal objects illuminated by white light. We obtain a resolution of about $0.4 \mathrm{~mm}$ in the green light of the $\mathrm{Hg}$ lamp, while for other wavelengths it is slightly larger, anyway within $20 \%$. As a result, a 3D distribution of spectral power is obtained, with a resolution of approximately $60 \times 30$ independent values in the spatial directions, corresponding to a field of view of $18 \times 10 \mathrm{~mm}$, independent wavelengths in the range $430 \mathrm{~nm}-620 \mathrm{~nm}$, with a resolving power of about 150 .

Another issue to be discussed is the way the slit is imaged onto the sensor for a given wavelength. Due to basic diffraction arguments, the slit image will not be a perfect straight line but will have a hyperbolic-like shape instead. ${ }^{18}$ This is shown in the Appendix (Fig. 10), where a measure of the $\mathrm{Hg}$ lines shows the spectral line shape, and is compared to the results of raytracing. Nevertheless, the distortion with respect to the straight line is of the same order or smaller than the linewidth, also for the optimally focused green line. This means that this effect can be neglected, at least in our system.

\section{Results and discussion}

Here we report the results obtained with the apparatus described earlier from samples with different spectral features. We only show a few images at given wavelengths, while more information is available in the online supplementary material, where the images are shown as short movies in which the different wavelengths are represented by the elapsing time. We stress that unfortunately we cannot test the results with an independent hyperspectral camera so that we actually quantify the resolution limits and uncertainties on the basis of a set of

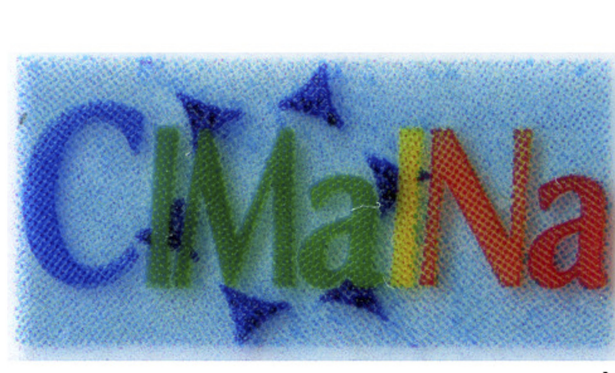

a)

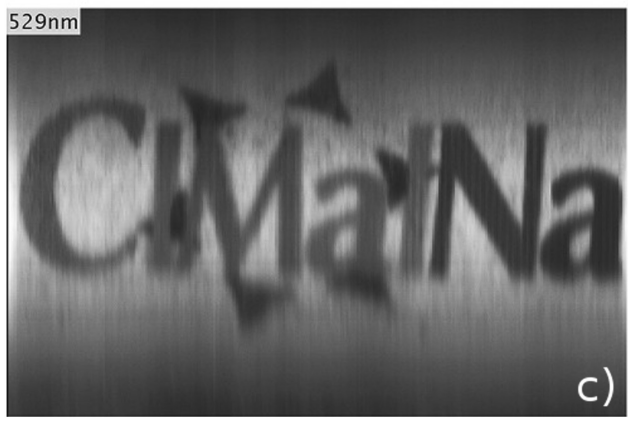

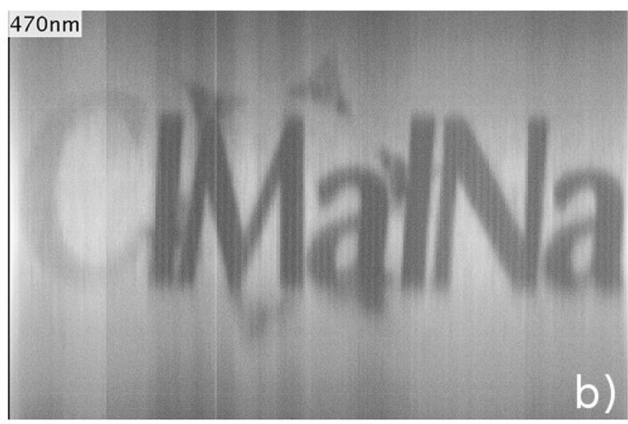

$600 \mathrm{~nm}$

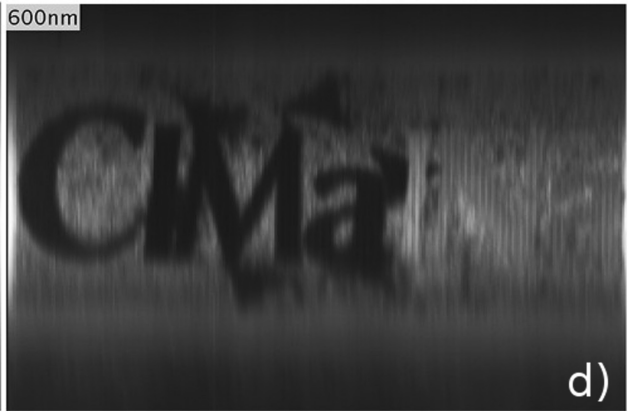

FIG. 5. (a) White light image of the sample, a transparency film with the writing CIMaINa printed on it with a CMYK laser printer. [(b)-(d)] Examples of three hyperspectral images. The wavelengths are indicated in the upper left corner of each image. 


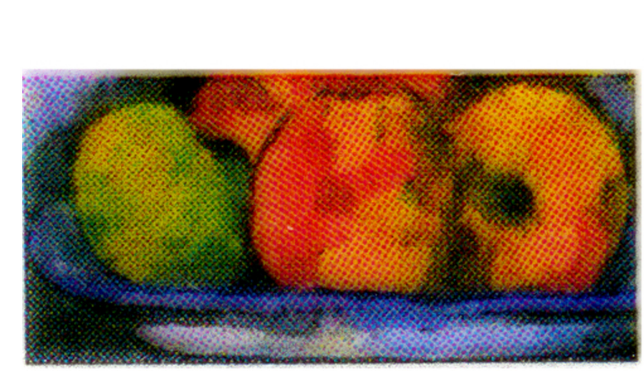

a)

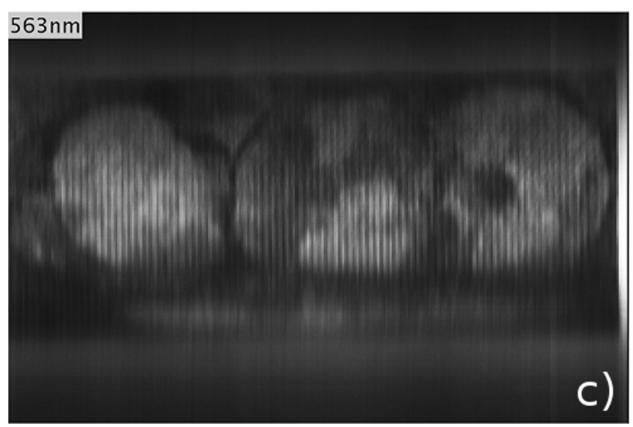

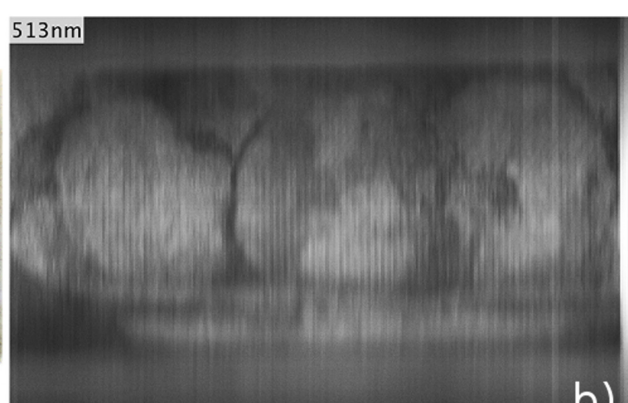

b)

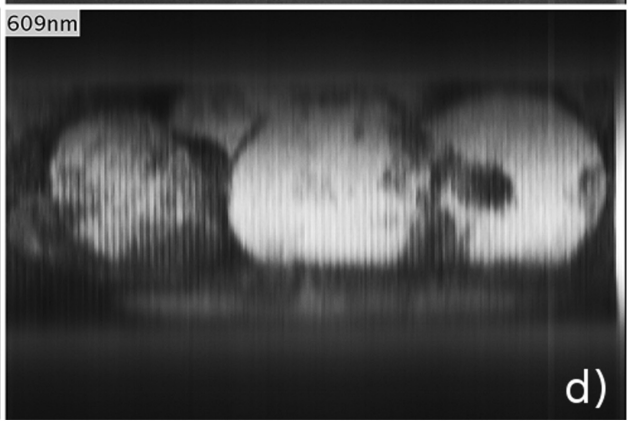

$609 \mathrm{~nm}$
FIG. 6. Hyperspectral imaging of a portion of the painting: Cezanne, Paul. Nature morte au tiroir ouvert. Oil on canvas, 1877-1879. Musée d'Orsay. The image was printed on a transparency film with a CMYK laser. Photograph by Jean-Pierre Dalbéra, used under the (CC BY 4.0) license (https://creativecommons.org/licenses/ by/4.0)https://commons.wikimedia.org/ wiki/File:Nature_morte_(Paul_C\%C3\% A9zanne)_(3332859798).jpg. ancillary measurements made on the same optical setup, as discussed in the Appendix. These measurements have been carefully supported by dedicated models, in order to provide more general rules to evaluate these parameters for any other optical realization of a system like this.

Figures 5 and 6 report images obtained at three representative wavelengths for samples constituted by color transparencies obtained with a laser printer. The color code is the CMYK, that is, the usual subtractive model. In both figures, the upper left panel reports "true" pictures of the samples obtained in reflected light with a white light illumination. Other panels show examples of intensity plots for three given wavelengths (as indicated in the upper left corner of each panel). Grey tone code is black for low intensity and white for high intensity.

Wavelengths have been chosen to evidence the peculiar behavior of the colors examined through the hyperspectral method. In particular, in Fig. 5, the logo and name of CIMaINa are analyzed. Data clearly show that blue and red are formed by almost pure cyan and magenta, respectively (they show maximum intensity at $470 \mathrm{~nm}$ and $600 \mathrm{~nm}$ ). On the contrary, green is composed by both cyan and yellow, with no magenta.

In Fig. 6, we report the results of hyperspectral imaging of a transparency prepared as the previous one, reproducing a detail of the Cezanne's painting "Still life with open drawer." The blue border of the dish is particularly interesting, compared to the white, lower part of it, which appears bright in the three wavelengths.

The picture in Fig. 7 has been obtained by transilluminating a small portion of an apple peel with small regions of different colors. The idea is to analyze a sample that is not limited by the CMYK printing pattern, which of course gives hyperspectral images limited to the colors imposed by the printer. The drawback here is that no independent check of the results is possible without a reference hyperspectral camera. HSI has been recognized as a very powerful technique for the measurement of quality attributes of fruits. ${ }^{19,20}$ Apples have been particularly studied with HSI in order to derive an automatic and rapid assessment of apple firmness, mealiness, and chilling defects. ${ }^{20}$ Different spectral behaviors of the apple peel are fingerprints of the presence of molecules such as chlorophyll or anthocyanins whose relative quantities can be correlated to the organoleptic properties of the fruit. $^{21}$

In Fig. 7, we show the apple and the region from which the peel has been taken off for the HSI analysis. Figure 8 shows the apple peel transilluminated with white light and three different images at three wavelengths. Blue images give very faint signals so that we concentrate on the yellow-red portion of the visible spectrum. It is evident that the bright yellow part in the upper right corner does contain appreciable red components, which would not be evident in the original picture.

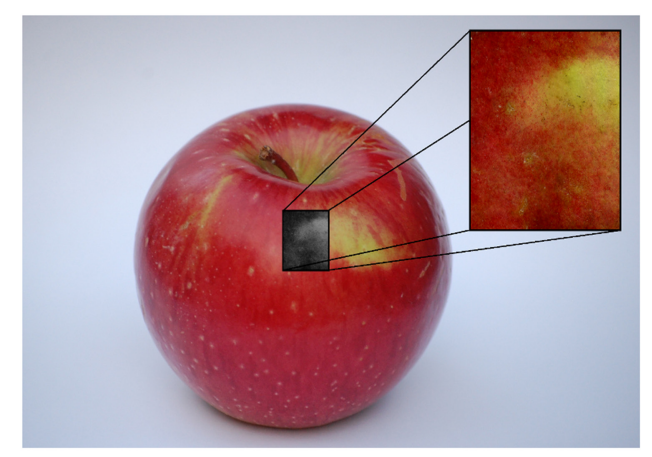

FIG. 7. Detail showing the fragment of the peel taken off from a red apple, which has been measured with our hyperspectral imaging apparatus. 

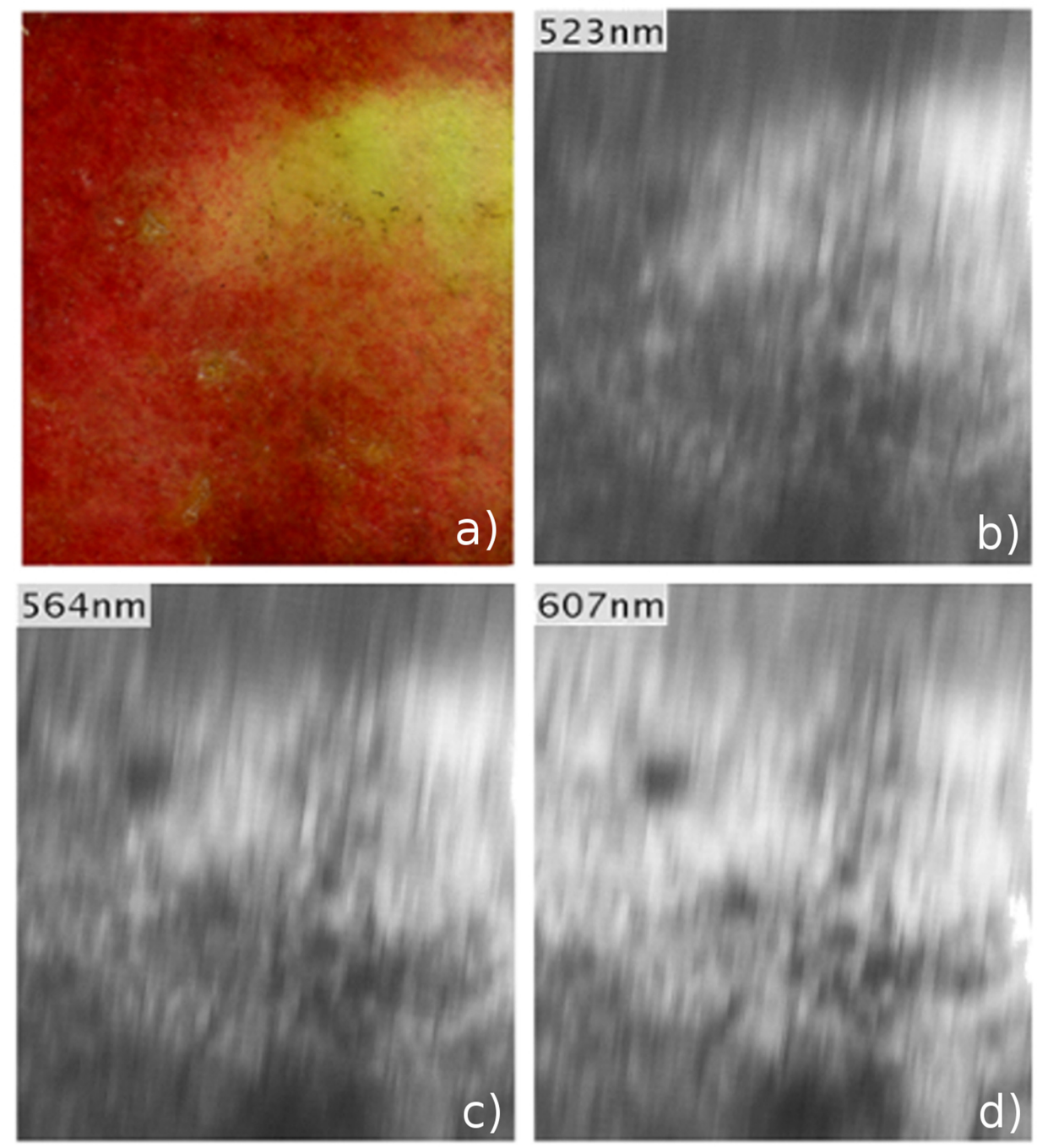

FIG. 8. Hyperspectral imaging of the fragment of a red and yellow apple peel. Here we show the wavelengths ranging from green to red across the visible spectrum.

\section{CONCLUSIONS}

In summary, we have shown the proof of the concept that adopting stretchable optics in a HSI device can result in a simplification of the apparatus, therefore overcoming the main difficulties currently preventing cheap and widespread applications of HSI sensors. A crucial role is played by the deformable diffraction grating, which gives both diffractive and optical powers by reflection, thus realizing a diffractive-catoptric optical device. This makes it easier to minimize aberrations that are essentially limited to the chromatic diffraction effects. Moreover, the choice to work with cylindrical surfaces has the advantage to keep the groove spacing of the grating unchanged. The current achievement of a hyperspectral camera having a resolving power of 150 represents a proof of the concept of several systems based on highly deformable polymeric gratings. The reflectivity of these components has been accurately studied in previous papers ${ }^{15,16}$ and sets around 0.6-0.7 in the visible range.

Current limitations are represented by the non-optimal surface quality, as discussed earlier, that gives rise to distortions in the diffracted wavefronts. Moreover, an active mount could compensate large scale deviations from the desired curvature of the surface. Despite the extended spectral range and the simplified optical scheme, it is possible to work with a traditional CCD sensor and achieve spectral resolution down to a few nanometers. Even if our system is still not reaching spatial resolution comparable to ordinary hyperspectral cameras, we think that the reduction of the number of optical elements represents a relevant step toward miniaturization and/or easy replication of these systems. Supersonic cluster beam implantation simplifies the fabrication process of deformable optical elements, which can reduce the overall cost of the device while ensuring stability and durability. There is a scope of improvement in the fabrication protocol of the PDMS gratings that can further enhance the quality of the hyperspectral images. For example, a dedicated photolithographic master for the gratings may be used to minimize the imperfections of the grooves.

Besides the usual applications of hyperspectral imaging, the possibility to realize relatively low cost, easily replicable spectral cameras may represent one of the best exploitations of such cameras in astronomy. The general trend of instrumentation for astronomy is toward multi objects ${ }^{22}$ and integral field spectroscopies, ${ }^{23}$ obtained by multiplexing instrumentation with thousands of multi-mode optical fibers to sample simultaneously many astronomical objects in view of large surveys aimed to study galaxies and exoplanets. Examples 
are VIRUS (30 000 fibers). ${ }^{24}$ The possibility to realize that a large number of identical, relatively simple instruments could support these applications in astronomy.

\section{SUPPLEMENTARY MATERIAL}

See supplementary material for short movies showing images of the samples (Figs. 5, 6, 8) where elapsing time represents the wavelength coordinate of the hyperspectral cube. Wavelength is displayed on the top right corner.

\section{APPENDIX: FOCUSING PROPERTIES OF THE GRATINGS AND PARAXIAL RAY-TRACING}

We provide here further information concerning the focusing properties of the deformable gratings by characterizing the intensity distribution of a plane wave in the far field. Moreover, we report the characterization of distortion and magnification of the optical system as a function of the wavelength. Spectral lines show a curved shape that can be accounted for.

\section{Quality of the focused wavefronts}

A careful characterization of the gratings focusing performances has been carried out. Specific measurements have been performed with a He-Ne laser beam spatially filtered and expanded to $10 \mathrm{~mm}$ in diameter, sent onto the grating. The grating is similar to the one described in Sec. II B, except for the radius of the cylindrical surface, $\mathrm{R}$, which has been imposed to be $130 \mathrm{~mm}$. In such a way, an astigmatic wavefront has been generated, thus separating the $\mathrm{V}$ and $\mathrm{H}$ focusing. Measurements have been performed along the direction of the diffracted beam, in order to measure the size variations of the beam due to the astigmatic wavefront modulation imposed by the grating. A distance of $40 \mathrm{~mm}$ around the two focuses has been spanned by taking images each of $1 \mathrm{~mm}$. The minimum size positions for the vertical and horizontal cases are in good accordance with the expected values obtained from Eqs. (1) and (2). Also, the convergence/divergence of the beam is in good accordance with what is expected for a Gaussian beam. Nevertheless, around both focal positions, the sizes of the beam do not decrease as expected, as the focusing ability is drastically limited. Using different gratings has given similar results, which are the object of further inspection.

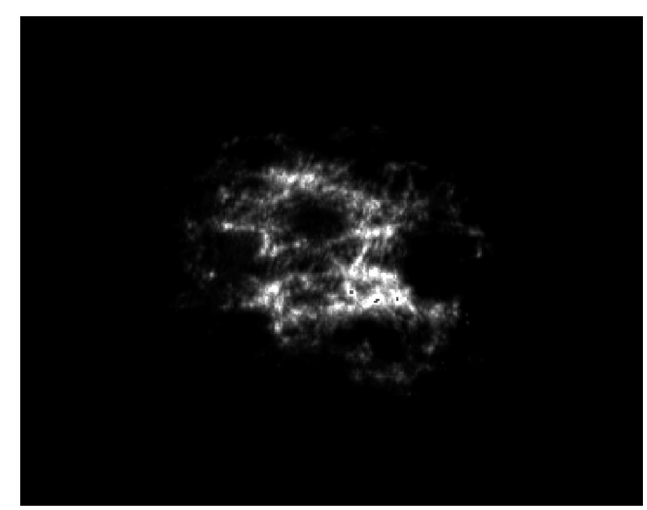

FIG. 9. Intensity distribution in the far field of the diverging beam after the focal position. The overall intensity profile is affected by fine structures, while the angular extension of the whole beam is in accordance with the Gaussian optics properties imposed by the horizontal and vertical curvatures.

First, by focusing the beam through a $f=100 \mathrm{~mm}$ positive lens, a focal spot of approximately $10 \mu \mathrm{m}$ has been obtained, which is close to the diffraction limit conditions. A similar condition should then be obtainable also for the astigmatic focuses of the diffracted beam.

Then, we studied the astigmatic wavefronts in the far field of the diffracted light. The result is reported in Fig. 9: a finely structured, inhomogeneous intensity distribution is present. The overall intensity profile is still imposed by the beam divergence, and it is in accordance with what is expected in terms of divergence. This confirms that the higher spatial frequencies (larger angles) imposed on the wavefront by the grating are behaving properly. By contrast, the intensity distribution is highly modulated on smaller lengthscales in the far field, meaning that wavefront aberrations over a large scale are imposed at the grating position. As a result, this proves that the beam diffracted by the grating is affected by extended modulations limiting the focusing properties.

An estimate of the ratio between the overall intensity distribution and the smallest speckle size is of the order of 50-100. This implies that the resolution of the optical system will be limited by the same factor due to the limited spatial coherence, bringing to a resolving power of the order of 200 for the groove spacing adopted here, $d=740 \mathrm{~nm}$. This is slightly smaller than the resolution obtained from our data.

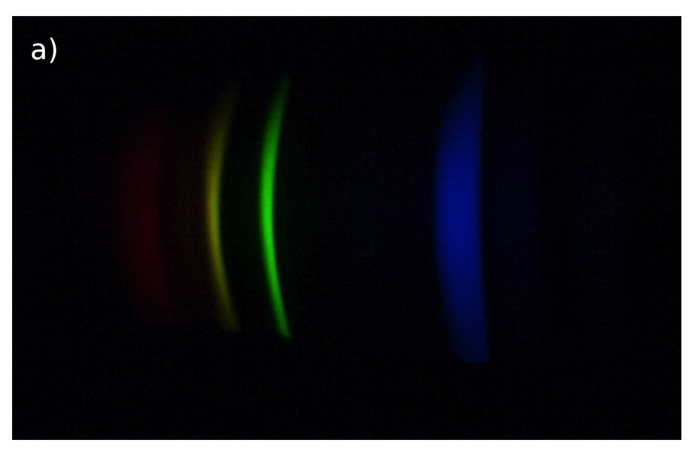

b)

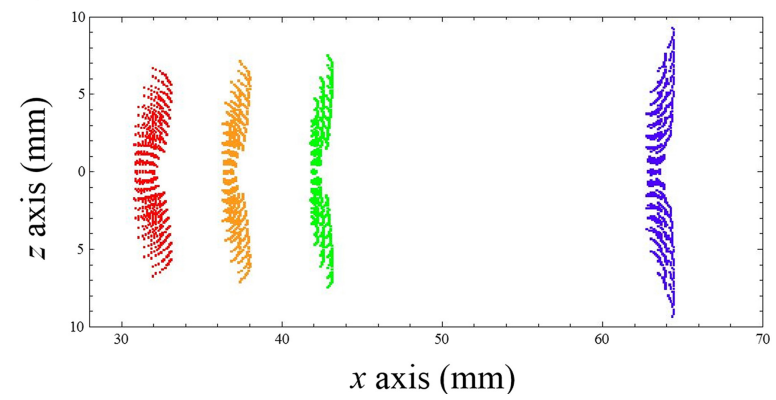

FIG. 10. (a) Slit image for the lines at different wavelengths of the low pressure $\mathrm{Hg}-\mathrm{Ar}$ lamp. (b) Ray-tracing results. 


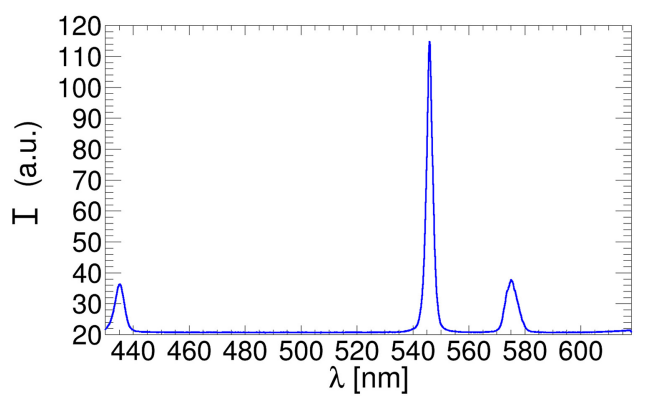

FIG. 11. Spectrum of the Hg lines obtained from the measurement in Fig. 10.

\section{Distortions of the slit images}

For a given wavelength, the system is not imaging the slit as a straight line, introducing a wavelength dependent distortion effect. As a result, we have modeled this distortion, in order to take it into account when recovering the images. Figure 10(a) represents the shapes of the slit images for different wavelengths [Fig. 10(a)] and the results of a dedicated code for ray-tracing calculated for exactly the geometry adopted in this work [Fig. 10(b); see below]. In Fig. 11, the intensity profile of this measurement is shown. As discussed in the text, distortions are small or at most comparable to the linewidth so that this effect has not been corrected for in this work.

However, this may not be the case if the curvature of the grooves is larger or even if the angular spread of the light emerging from the slit is larger. In order to give a description of this effect and to quantitatively evaluate it under arbitrary realizations of the apparatus, we realized a fully analytical model of the rays impinging off-axis and then diffracted by the curved grating with circular grooves, thus exactly reproducing our optical system. Here we report the results of the off axis rays that impinge onto the grating surface after being refracted by the lens after the slit. Due to the specific illumination, light is strongly forward directed, thus appreciably reducing the angular spread of the light (i) entering the lens and (ii) impinging onto the grating. This makes the system working with rays that propagate appreciably off-axis in the vertical direction, with a considerable effect in distorting the spectral lines. In general, the reduction of the angular spread would reduce the spectral and geometrical (vertical) resolution of the optical system. Nevertheless, due to the limited performances demanded to the system, we maintain the resolution properties described earlier (and experimentally proved). In Fig. 10(b), we report the results of the raytracing obtained by impinging the grating with rays spread over a regular array of points spanning overall the illuminated region (approximately $8 \times 14 \mathrm{~mm}^{2}$ ). Angles are imposed to cover a spread of $0.06 \mathrm{rad}$ around the directions of the rays emerging by the lens illuminated by parallel beams from the slit.

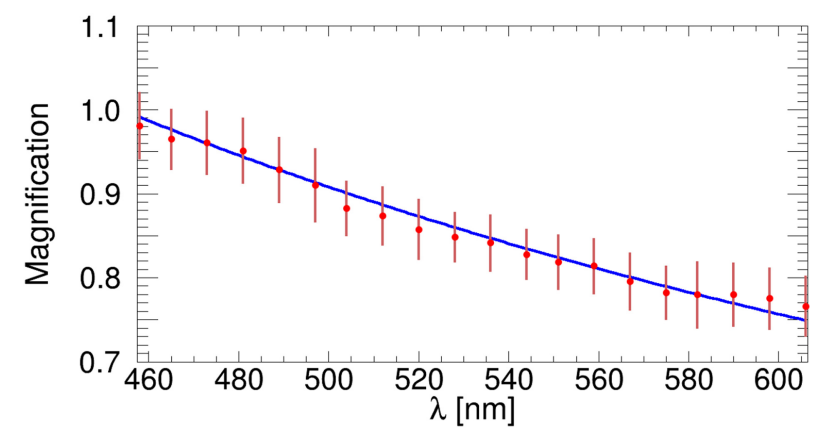

FIG. 12. We show the magnification as a function of the wavelength. The red dots correspond to the measured values, each of which has its corresponding uncertainty displayed as an error bar. The blue solid line is the expected magnification, deduced from Eqs. (3) and (4).

\section{Magnification}

By imaging a sample with known size and comparing it to its image, we measure the magnification in the vertical direction. In Fig. 12, the magnification in the vertical direction is shown as a function of the wavelength. It depends on the wavelength just due to the change of focal distance of the grating. This allows for a simple evaluation of the magnification.

${ }^{1}$ D. Bannon, Nat. Photonics 3, 627 (2009).

${ }^{2}$ J. Gilchrist, Hyperspectral Imaging Spectroscopy: A Look at Real-Life Applications, Photonics Handbook (Lauren Publishing, 2007).

${ }^{3}$ Y. Z. Feng and D. W. Sun, Crit. Rev. Food Sci. Nutr. 52(11), 1039 (2012).

${ }^{4}$ G. Lu and B. Fei, J. Biomed. Opt. 19(1), 010901 (2014).

${ }^{5}$ C. P. Warren, D. Even, W. Pfister, K. Nakanishi, A. Velasco, D. Breitwieser, S. Yee, and J. Naungayan, Opt. Eng. 51(11), 111720 (2012).

${ }^{6}$ P. Pottier and M. Packirisamy, Appl. Opt. 51, 4073 (2012).

${ }^{7}$ C. N. Brown, Ann. Sci. 72(1), 28 (2015).

${ }^{8}$ J. James, Spectrograph Design Fundamentals (Cambridge University Press, Cambridge, 2007).

${ }^{9}$ M. Czerny and A. F. Turner, Z. Phys. 61, 792 (1930).

${ }^{10}$ M. C. Hutley, in Diffraction Gratings, edited by N. H. March and H. N. Daglish (Academic Press, 1982).

${ }^{11}$ R. A. Guerrero, M. W. C. Sze, and J. R. A. Batiller, Appl. Opt. 49(19), 3634 (2010).

${ }^{12}$ J. Ihlemann, S. Müller, S. Puschmann, D. Schäfer, M. Wei, J. Li, and P. R. Herman, Appl. Phys. A: Mater. Sci. Process. 76(5), 751 (2003).

${ }^{13}$ S. Shian, R. M. Diebold, and D. R. Clarke, Opt. Express 21(7), 8669 (2013).

${ }^{14}$ J. L. Wilbur, R. J. Jackman, G. M. Whitesides, E. L. Cheung, L. K. Lee, and M. G. Prentiss, Chem. Mater. 8(7), 1380 (1996).

${ }^{15}$ C. Ghisleri, M. Siano, L. Ravagnan, M. A. C. Potenza, and P. Milani, Laser Photonics Rev. 7(6), 1020 (2013).

${ }^{16}$ C. Ghisleri, M. A. C. Potenza, L. Ravagnan, A. Bellacicca, and P. Milani, Appl. Phys. Lett. 104(6), 061910 (2014).

${ }^{17}$ S. Weinberg, Gravitation and Cosmology (Wiley, New York, USA, 1972).

${ }^{18} \mathrm{~J}$. W. Goodman, Introduction to Fourier Optics (McGraw-Hill, New York, USA, 1968).

${ }^{19}$ J. Qin and R. Lu, Postharvest Biol. Technol. 49(3), 355 (2008).

${ }^{20}$ H. Huang, L. Liu, and M. O. Ngadi, Sensors 14(4), 7248 (2014).

${ }^{21}$ G. ElMasry, N. Wang, and C. Vigneault, Postharvest Biol. Technol. 52(1), 1 (2009).

${ }^{22}$ J. M. Hill, J. R. P. Angel, J. S. Scott, and D. Lindley, Astrophys. J. 242, L69 (1980).

${ }^{23}$ C. Vanderriest, Publ. Astron. Soc. Pac. 92, 858 (1980).

${ }^{24}$ G. J. Hill, P. J. MacQueen, P. Palunas, A. Kelz, M. M. Roth, K. Gebhardt, and F. Grupp, New Astr. Rev. 50, 378 (2006). 